\title{
Clitoral Enlargement Secondary to Neurofibromatosis
}

Wilfred S. Kearse Jr., M.D.

Michael L. Ritchey, M.D.

\section{Introduction}

$\mathrm{F}$ amilial neurofibromatosis, or von Recklinghausen's disease, was first described more than 100 years ago. Characteristic findings are pigmented skin lesions (café-au-lait spots) and soft-tissue tumors arising from the neural sheath in any part of the body. Genitourinary neurofibromatosis is uncommon, with little more than 50 reported cases of bladder involvement. ${ }^{1}$ Even more rare is genital involvement, which can present clinically as ambiguous genitalia in the female patient. We herein report a case of clitoromegaly secondary to a localized neurofibroma and review the diagnostic features and management of this unusual problem.

\section{Patient Report}

An 8-year-old girl was previously discovered to have neurofibroma-

From the Department of Urology Joint Military Medical Command Wilford Hall

USAF Medical Center

San Antonio, Texas

Address correspondence to: Michael Ritchey, M.D., Department of Surgery, Section of Urology, University of Michigan, 1500 E. Medical Center Drive, Ann Arbor, MI 48109-0330

The views expressed are those of the authors and do not reflect the opinions of the United States Air Force or the Department of Defense. tosis during evaluation for thoracic scoliosis. The child's mother had previously been diagnosed with neurofibromatosis. Urologic consultation was obtained for the girl to evaluate new-onset diurnal and nocturnal enuresis. On physical examination, she was noted to have multiple café-au-lait spots but was otherwise normally developed. She had a prepubertal female habitus, but examination of the genitalia revealed an enlarged clitoris (Figure 1). Significantly, the glans clitoris was soft to palpation, while the clitoral shaft was very firm and nodular. Endocrine consultation was obtained. Her karyotype was $46, \mathrm{XX}$. Laboratory values, including serum electrolyte, adrenocorticotropic hormone (ACTH), cortisol, pregnanetriol, and urinary ketosteroid concentrations, were all normal. Urologic evaluation included an intravenous pyelogram and voiding cystourethrogram, which were both normal. Urodynamic evaluation with a cystometrogram demonstrated uninhibited detrusor contractions with a decreased functional capacity. Imaging of the thoracic spine revealed a paraspinous neurofibroma without any cord involvement. She was able to void spontaneously with minimal residual urine and was started on oxybutynin, with resolution of her enuresis.

During the next year, she had further enlargement of the clitoris and it was elected to perform clitoral reduction. At operation, a 3- $\times 1-\mathrm{cm}$ mass was found adhering to the corporal bodies. The mass was excised with preservation of the neurovascular bundle, and the glans clitoris was recessed. The pathologic diagnosis was a plexiform neurofibroma. There is no evidence of recurrence one year later.

\section{Discussion}

Genitourinary involvement with neurofibromatosis is uncommon. Lesions of the genitourinary system are most commonly derived from the pelvic autonomic plexus. Symptoms of lower urinary tract involvement include frequency, urgency, hematuria, and difficulty voiding. Rectal exam or palpation of the suprapubic region frequently reveals a mass. Bladder capacity may be reduced, with poor vesical compliance. About $20 \%$ of cases involving the bladder have simultaneous genital involvement. ${ }^{1}$

Nine pediatric cases of clitoral involvement by neurofibromatosis have been reported ${ }^{1-9}$ (Table 1 ). Five of these children had an associated lesion of the genitourinary tract: three with labial involvement, one with labial and bladder involvement, and one with bladder and vaginal involvement. The clinical presentation of these young girls was ambiguity of the genitalia due to the considerable clitoral involvement. Simultaneous involvement of the labia can give the appearance of a labioscrotal gonad. 


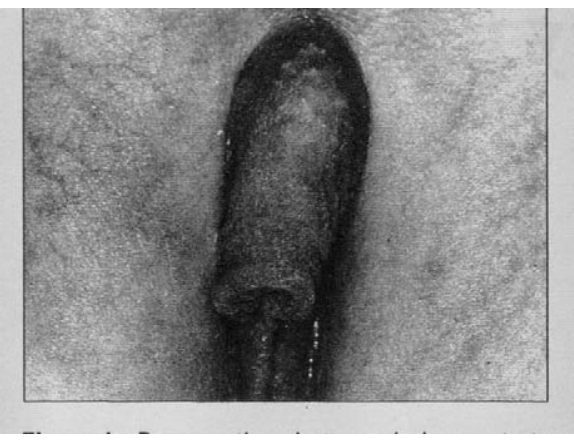

Figure 1. Preoperative photograph demonstrating clitoromegaly secondary to the neurofibroma.

Plexiform neurofibromas are the most common histologic type involving the genitalia. Microscopically, the tumor is formed by the combined proliferation of all the elements of a peripheral nerve: axons, Schwann cells, fibroblasts, and, probably, perineural cells. Schwann cells are believed to be the cells of origin. ${ }^{10}$ Malignant transformation of neurofibromas for all sites has a quoted incidence of $13 \%$ to $29 \% .^{3}$

The preferred treatment is to perform clitoroplasty after removal of the neurofibroma. Resection of the clitoris should be reserved for diffuse involvement of the corpora preventing simple local excision. These tumors are generally not well encapsulated, which can make excision more difficult. Surgical intervention has been thought to stimulate sarcomatous change, and local recurrence has been a problem following excision of neurofibromas in other sites. Local recurrence or malignant degeneration following excision of neurofibromas involving the clitoris has not been reported, but follow-up has been limited.

Neurofibroma should be considered in the differential diagnosis of any child who presents with idiopathic clitoromegaly or other genital masses. Careful examination for other physical manifestations of neurofibromatosis should be undertaken, along with a di-

Table 1

\section{REPORTED CASES OF CLITORAL INVOLVEMENT WITH NEUROFIBROMATOSIS}

Author
Rink et al
Labardini et a
Haraoka et al
Kanetiet al
Ravi
Schs
Gres
Hads
Ken:
Pres:
GU:

rected family history. The striking feature on physical examination of the genitalia is the nodularity and firmness of the clitoris. Because of the association of bladder involvement in patients with neurofibromas of the clitoris, a complete evaluation of the urinary tract is warranted. The workup should include an intravenous urogram and voiding cystourethrogram, along with cystoscopy and biopsy of suspicious areas, if warranted. Children with significant deformity should be offered surgical treatment. Reconstructive procedures of the genitalia, such as clitoral reduction, should be possible in most cases.

\section{REFERENCES}

1. Rink RC, Mitchell ME. Genitourinary neurofibromatosis in childhood. JUrol. 1983;130:1176-1179.

2. Labardini MM, Kallet HA, Cerny JC. Urogenital neurofibromatosis simulating an intersex problem. $J$ Urol. 1968;98:627-632.
3. Haraoka M, Naito S, Kumazawa J. Clitoral involvement by neurofibromatosis: a case report and review of the literature. J Urol. 1988;139:95-96.

4. Kaneti J, Lieberman E, Moshe P, et al. A case of ambiguous genitalia owing to neurofibromatosis - review of the literature. JUrol 1988;140:584585.

5. Ravikumar VR, Lakshmanan D. A solitary neurofibroma of the clitoris masquerading as intersex. $J$ Pediatr Surg. 1983;18:617.

6. Schepel SJ, Tolhurst DE. Neurofibromata of clitoris and labium majus simulating a penis and testicle. Bril J Plast Surg. 1981;34:221-223.

7. Greer DM Jr, Pederson WC. Pseudomasculinization of the phallus. Plast Reconstr Surg. 1981;68:787-788.

8. Haddad HM, Jones HW Jr. Clitoral enlargement simulating pseudohermaphroditism. J Dis Child. 1960;99:282-287.

9. Kenny FM, Fetterman GH, Preeyasombat C. Neurofibromata simulating a penis and labioscrotal gonads in a girl with von Recklinghausen's disease. Pediatrics. 1966;37:456-459.

10. Rosai J. Soft tissues. In: Rosai J, ed. Ackerman's Surgical Pathology. 7th ed. St. Louis, MO: CV Mosby Co; 1989:1567-1568. 